\title{
WATER-SOLUBLE ANTIOXIDANT IN EWES DURING THEIR LATE PREGNANCY
}

\author{
B. Fürll ${ }^{1}, H$. Wilken², M. Fürll ${ }^{2}$ \\ ${ }^{1}$ Institute of Veterinary Physiology, ${ }^{2}$ Department of Internal Medicine, \\ Veterinary Faculty Leipzig, e-mail: mfuerll@rz.uni-leipzig.de
}

Objective: The metabolism of ewes pregnant with several foetuses is often severely stressed during the end of the pregnancy, which may cause pregnancy toxaemia. These disturbances effect primarily the energy metabolism, but also parameters such as albumin and bilirubin, which again influence the antioxidative state. The antioxidative state also depends from the supply with antioxidants by foodstuffs. Our project aimed at checking the antioxidative state in Merino-meat-sheep and Black-headsheep pregnant with one or two foetuses during their late pregnancy compared with other metabolic parameters.

Experimental design: Sonographically, 4 healthy Merino-meat-sheep pregnant with one (MFS 1) and pregnant with two (MFS2) foetuses as well as 4 twin pregnant Black-head-sheep with (S K2) were selected. Weekly blood samples (V. jugularis) were taken during the last five weeks ante partum (w. a. p.) as well as during the second week post partum (w. p. p.). The oxidative state was determined as a trolox equivalent antioxidative capacity (TEAC). Hitachi 705 as well as Technicon 1 were used to metabolic and haematological analyses.

Results: Ante and post partum, TEAC concentrations were lowest in MFS1 compared with MFS2 as well as S K2 $(\mathrm{p}<0.05)$. Tendentially, the concentration decreased in all groups 3 to $1 \mathrm{w}$. a. $\mathrm{p}$. The TEAC differences between the groups, can be explain as a result of differing food intake. The following table shows the quartiles values (median, first -, third quartiles) of different parameters:

\begin{tabular}{|c|c|c|c|c|c|}
\hline \multirow{2}{*}{$\begin{array}{l}\text { para- } \\
\text { meters }\end{array}$} & \multirow[b]{2}{*}{ race } & \multicolumn{4}{|c|}{ weekspostpartum } \\
\hline & & -5 & -3 & -1 & 2 \\
\hline TEA & MFS 1 & 180 & $171 ; 155 ; 188$ & $179 ; 175 ; 184$ & 175; \\
\hline \multirow[t]{2}{*}{$\mathrm{C} \mu \mathrm{mol} / \mathrm{l}$} & MFS 2 & $193 ; 189 ; 197$ & $194 ; 178 ; 198$ & $184 ; 178 ; 185$ & $214 ; 194 ; 215$ \\
\hline & S K 2 & $180 ; 170 ; 187$ & $190 ; 180 ; 201$ & $190 ; 185 ; 200$ & $184 ; 166 ; 201$ \\
\hline \multirow{3}{*}{$\begin{array}{l}\text { Albu- } \\
\text { mine g/l }\end{array}$} & MFS 1 & $36.7 ; 35.8 ; 37.5$ & $40.5 ; 40.0 ; 41.6$ & $40.9 ; 40.2 ; 42.4$ & $43.6 ; 41.5 ; 44.8$ \\
\hline & MFS 2 & $38.8 ; 38.8 ; 42.7$ & $38.9 ; 35.6 ; 39.4$ & $39.5 ; 36.5 ; 44.6$ & $38.9 ; 34.9 ; 40.7$ \\
\hline & S K 2 & $40.2 ; 37.0 ; 40.2$ & $41.7 ; 36.6 ; 43.2$ & $40.3 ; 38.5 ; 41.3$ & $41.2 ; 39.2 ; 43.3$ \\
\hline \multirow{3}{*}{$\begin{array}{l}\text { Biliru- } \\
\text { bine } \mu \mathrm{mol} / 1\end{array}$} & MFS 1 & $2.7 ; 2.2 ; 3.1$ & $2.8 ; 2.6 ; 3.3$ & $2.0 ; 2.4 ; 3.4$ & $3.8 ; 2.9 ; 4.2$ \\
\hline & MFS 2 & $3.1 ; 2.4 ; 3.2$ & $3.5 ; 3.1 ; 4.3$ & $3.8 ; 3.1 ; 4.8$ & $3.9 ; 2.6 ; 5.9$ \\
\hline & S K 2 & $2.3 ; 2.1 ; 2.7$ & $2.6 ; 1.3 ; 3.2$ & $2.2 ; 2.0 ; 2.7$ & $2.4 ; 1.8 ; 3.0$ \\
\hline
\end{tabular}

Albumin (0.47) and bilirubin (0.34) correlated significantly with TEAC, other parameters, such as insuline (-0.25), FFA (0.22), haemoglobin (0.20), total protein $(0.06)$, glucose $(0.05)$, BHB (0.02), monocytes $(-0.16)$ or leukocytes $(0.03)$, did not.

Conclusions: During late pregnancy different TEAC concentrations in single and twin pregnant ewes occurred, indicating of the antioxidative state. Their cause may be related to the food intake. Metabolic influences (albumin : bilirubin) have in part opposite effects. 